\title{
INDICADORES DO PERFIL LIPÍDICO PLASMÁTICO RELACIONADOS À RESISTÊNCIA À INSULINA
}

\author{
Ana Carolina Junqueira Vasques*1, lina Enriqueta Frandsen Paez de Lima Rosado², Gilberto Paixão Rosado², Rita de Cassia Lanes Ribeiro², \\ Sylvia do Carmo Castro Franceschini², Silvia Eloiza Priore², Bruno Geloneze ${ }^{3}$, Dirce Ribeiro de Oliveira ${ }^{4}$ \\ Trabalho elaborado na Universidade Federal de Viçosa - Departamento de Nutrição e Saúde
}

*Correspondência:

Laboratório LIMED -

Gastrocentro

Rua Carlos Chagas, 420,

Cidade Universitária

"Zeferino Vaz".

Barão Geraldo

CEP 13031-970

Campinas - SP

\section{RESUMO}

ОвJEтIvos. Investigar a habilidade de indicadores bioquímicos do perfil lipídico plasmático em identificar resistência à insulina (RI), avaliada pelo índice HOMA-IR (Homeostasis Model Assessment - Insulin Resistance).

MÉTodos. Foram avaliados 138 homens saudáveis (20-59 anos). Analisaram-se os seguintes indicadores bioquímicos do perfil lipídico: triglicérides (TG), colesterol total (CT), HDL-C, LDL-C, e as relações CT/HDL-C e TG/HDL-C. Considerou-se o percentil 75 como ponto de corte para o índice HOMA-IR. A análise estatística constou do cálculo do coeficiente de correlação de Spearman e da construção de curvas ROC, com o cálculo das áreas abaixo da curva (AUC).

Resultados. A relação TG/HDL-C $(r=0.334$ e AUC $=0.724 \pm 0.046 p<0.001)$ apresentou a correlação mais forte e a maior AUC, respectivamente, seguida do HDL-C ( $r=-0,313$ e AUC $=0,716 \pm$ $0.052, p<0.01)$, dos TG $(r=0.261$ e AUC $=0.674 \pm 0,048 ; p<0,01)$ e da relação CT/HDL-C $(r$ $=0.259$ e AUC $=0.655 \pm 0.05, p<0.01)$. $0 \mathrm{CT}$ e o LDL-C não apresentaram resultados estatísticos significantes $(p>0,05)$.

ConcLusão. A relação TG/HDL-C apresentou melhor habilidade em identificar RI, representando um instrumento alternativo e de fácil acesso para a avaliação da RI na prática clínica, proporcionando intervenções de caráter preventivo de doenças na população do sexo masculino.

UnITERMOS: Resistência à insulina. HOMA. Lipoproteínas. Triglicérides.

\section{INTRODUÇÃO}

A resistência à insulina $(\mathrm{RI})$ representa uma alteração metabólica de etiologia genética e ambiental caracterizada por resposta anormal dos tecidos periféricos à ação da insulina circulante ${ }^{1}$. Ela tem sido considerada a base fisiopatológica entre as diversas alterações presentes na síndrome metabólica, como a hipertensão arterial, a intolerância à glicose, o diabetes, as dislipidemias e a presença de estados pró-inflamatórios².

A técnica de clamp euglicêmico hiperinsulinêmico representa o melhor método para a determinação da RI. Contudo, por ser dispendiosa e altamente invasiva, ela é utilizada apenas na pesquisa clínica. O índice HOMA-IR (Homeostasis Model Assessment of Insulin Resistance) é uma das alternativas para a avaliação da RI nos estudos epidemiológicos, por ser de aplicação mais fácil e por apresentar correlação forte com o clamp $p^{3,4}$. A identificação de um instrumento acessível e alternativo para a determinação da RI na prática clínica tem se tornado uma necessidade, pois viabilizaria intervenções precoces, além de melhor avaliação e melhor acompanhamento dos pacientes.
Trabalhos realizados com caucasianos ${ }^{5-8}$, com índios asiáticos e com mexicanos americanos ${ }^{9}$ identificaram bom desempenho dos TG e da relação triglicérides/HDL-C (TG/HDL-C) na predição de RI. Todavia, um bom desempenho para estes mesmos indicadores não foi identificado em americanos de origem africana ${ }^{10}$ e em negros não-hispânicos ${ }^{11}$, o que sugere comportamento distinto desses indicadores para populações de etnias diferentes.

A população brasileira, formada por uma miscigenação entre ameríndios, europeus e africanos, é uma das mais heterogêneas do mundo ${ }^{12}$, o que agrega características próprias à mesma. Dada a relativa facilidade de obtenção dos exames de perfil lipídico na rotina clínica e a grande heterogeneidade étnica da população brasileira, o presente estudo objetivou: 1) avaliar o comportamento dos indicadores bioquímicos do perfil lipídico em relação ao índice HOMA-IR e 2) investigar a habilidade dos indicadores bioquímicos do metabolismo das lipoproteínas plasmáticas em predizer RI em homens brasileiros aparentemente saudáveis.

\footnotetext{
1. Nutricionista - Doutoranda em Clínica Médica - LIMED - Laboratório de Investigação em Metabolismo e Diabetes - Gastrocentro, Universidade Estadual de Campinas. Campinas, SP

2. Professor Adjunto - Departamento de Nutrição e Saúde da Universidade Federal de Viçosa . Viçosa, MG

3. Pesquisador e coordenador do LIMED - Laboratório de Investigação em Metabolismo e Diabetes - Gastrocentro, Universidade Estadual de Campinas. Campinas, SP

4. Professor Adjunto - Escola de Enfermagem da Universidade Federal de Minas Gerais. Belo Horizonte, MG
} 


\section{Métodos}

Foi realizado um estudo transversal, no qual foram avaliados homens adultos saudáveis vinculados à Universidade Federal de Viçosa (UFV). Os dados foram coletados na Divisão de Saúde da UFV, localizada em Viçosa (MG), Brasil. O protocolo do estudo foi aprovado pelo Comitê de Ética em Pesquisa com Seres Humanos da UFV.

Os critérios de exclusão do estudo foram: glicemia de jejum $>99 \mathrm{mg} / \mathrm{dL}$, níveis de LDL-C $\geq 160 \mathrm{mg} / \mathrm{dL}$, triglicérides $\geq$ $150 \mathrm{mg} / \mathrm{dL}$, história prévia de evento cardiovascular, presença de hipertensão arterial e uso de medicamentos com efeitos no metabolismo dos carboidratos e dos lipídeos.

A pressão arterial foi aferida seguindo as normas propostas pela Sociedade Brasileira de Cardiologia ${ }^{12}$. A estatura foi determinada com a utilização de um estadiômetro fixo na parede e o peso foi aferido em balança eletrônica digital. Ambas as medidas foram realizadas segundo as técnicas preconizadas por Jelliffe ${ }^{13}$. A partir dos valores de peso e altura calculou-se o índice de massa corporal (IMC). Foram classificados com excesso de peso corporal aqueles que apresentaram valores de IMC $\geq 25 \mathrm{~kg} / \mathrm{m}^{214}$.

Nas análises bioquímicas foram determinados: glicose e insulina plasmáticas de jejum, colesterol total, HDL, LDL e triglicérides. As amostras de sangue foram coletadas após um jejum noturno de 12 horas. As determinações plasmáticas dos triglicérides, colesterol total, HDL-C e glicose foram realizadas pelo método colorimétrico enzimático. A fração LDL-C foi determinada segundo a fórmula de Friedwald ${ }^{15}$. As relações CT/HDL-C e TG/ HDL-C foram calculadas. A insulina plasmática foi determinada pelo método ELISA, com a utilização de kits ultrassensíveis e coeficientes de variação intraensaio e interensaio de 5,96 \pm $1,17 \mu \mathrm{U} / \mathrm{mL}$ e $10,3 \pm 0,9 \mu \mathrm{U} / \mathrm{mL}$, respectivamente. 0 método utilizado para a avaliação da RI foi o índice HOMA-IR (Homeostasis Model Assessment - Insulin Resistance),calculado a partir da fórmula ${ }^{16}$ : HOMA-IR = IJ (mU/L)x GJ (mmol/L)/ 22,5, onde IJ corresponde à insulinemia de jejum e GJ à glicemia de jejum. Considerou-se como ponto de corte para as análises o valor referente ao percentil 75 do HOMA-IR na amostra avaliada.

As análises estatísticas foram realizadas nos programas SPSS 12.0 e MedCalc 9.3. O nível de significância adotado foi inferior a $5 \%$ ( $p<0,05)$. Para as variáveis com distribuição normal, utilizou-se a análise de variância com o teste post-hoc de Tukey e o cálculo do coeficiente de correlação de Pearson. Para as variáveis que não apresentaram distribuição normal, empregou-se o teste de Kruskall-Wallis com o teste post-hoc de Dunn's e o cálculo do coeficiente de correlação de Spearman. Foram construídas curvas ROC (Receiver Operating Characteristic Curve) e calculadas as áreas abaixo das curvas, com um intervalo de confiança de $95 \%{ }^{17}$. O teste $Z$ foi utilizado para comparação das áreas abaixo das curvas.

\section{Resultados}

Foram avaliados 138 homens saudáveis com idade entre 20 e 59 anos. A caracterização dos participantes da pesquisa, realizada segundo os quartis de HOMA-IR, pode ser vista na Tabela 1. Destaca-se a uniformidade da idade seguindo os quartis do HOMA-IR. As variáveis IMC, pressão arterial diastólica, glicemia, insulinemia, TG e relação TG/HDL-C mostraram aumento e o HDL-C apresentou redução com o aumento do índice HOMA-IR.

Nas análises de correlação entre os indicadores bioquímicos do perfil lipídico e os valores de HOMA-IR, com exceção do CT e do LDL-C, todas as correlações foram altamente significantes $(p<0,001)$. A maior correlação foi para a relação TG/HDL-C ( $r=0,33)$, seguida do HDL-C isolado $(r=-0,31)$, dos TG $(r=0,26)$ e da relação CT/HDL-C $(r=0,26)$. O CT e o LDL-C não apresentaram correlações significantes ( $p>0,05)$.

De forma coerente com as análises de correlação, nas curvas ROC (Figura 1), a relação TG/HDL-C, seguida do HDL-C, apresentaram as maiores áreas abaixo da curva, e, consequentemente, a melhor habilidade para a avaliação de níveis mais elevados de HOMA-IR, comparados aos demais indicadores bioquímicos estudados (Tabela 2). O CT e o LDL-C apresentaram a menor eficácia, uma vez que suas áreas abaixo da curva foram $\leq 0,5$. Os resultados do teste $Z$ de comparações múltiplas dois a dois identificaram que as áreas abaixo da curva para o HDL-C foram maiores que as áreas formadas para o CT $(p=0,039)$ e para o LDL-C ( $p=0,003)$. Para os TG, a área abaixo da curva foi maior que a do LDL-C ( $p=0,016)$, enquanto a área da relação $T G / H D L-C$ foi superior às áreas formadas para o LDL-C $(p=0,003)$ e CT $(p=0,015)$.

\section{Discussão}

Os principais achados do presente estudo demonstram que alguns indicadores bioquímicos do perfil lipídico podem ser considerados instrumentos alternativos para a avaliação de RI. Entre os indicadores metabólicos avaliados, a relação TG/HDL-C, seguida do HDL-C, foram os que apresentaram maior correlação com o HOMA-IR e melhor eficácia na análise ROC, enquanto que as dosagens de CT e de LDL-C não apresentaram resultados com significância estatística.

Bonora et al. ${ }^{18}$ identificaram maiores ocorrências de RI nos indivíduos hipertrigliceridêmicos ou naqueles com baixos níveis de HDL-C ( $85 \%)$ que nos indivíduos hipercolesterolêmicos ( $\sim 53,5 \%)$, sugerindo que esta última alteração metabólica está menos associada à RI em relação às demais. Em concordância, Oliveira et al. ${ }^{19}$ identificaram maiores níveis de HOMA-IR nos indivíduos com hipertrigliceridemia, embora o mesmo não tenha sido observado pra o HDL-C.

Os resultados do presente estudo foram coerentes com trabalhos realizados em etnias diferentes ${ }^{5-8}$, com exceção do HDL-C, que apresentou melhor eficácia em nosso estudo. Tal fato pode ser atribuído à presença de indivíduos com baixos valores de HDL-C na amostra e à ausência de indivíduos com níveis elevados de TG, o que pode ter enfraquecido as correlações para os TG e também ter influenciado na área abaixo da curva ROC para esta variável.

Um estudo conduzido com indivíduos caucasianos, em sua maioria, encontrou correlações mais fortes para os mesmos indicadores bioquímicos avaliados no presente estudo, sendo que a relação TG/HDL-C também foi o indicador de RI que apresentou correlação mais forte e maior área abaixo da curva na análise $\mathrm{ROC}^{6}$. Provavelmente, a maior força das correlações encontradas por estes autores pode ser explicada pelo maior tamanho da amostra avaliada e também pela maior amplitude de variação 


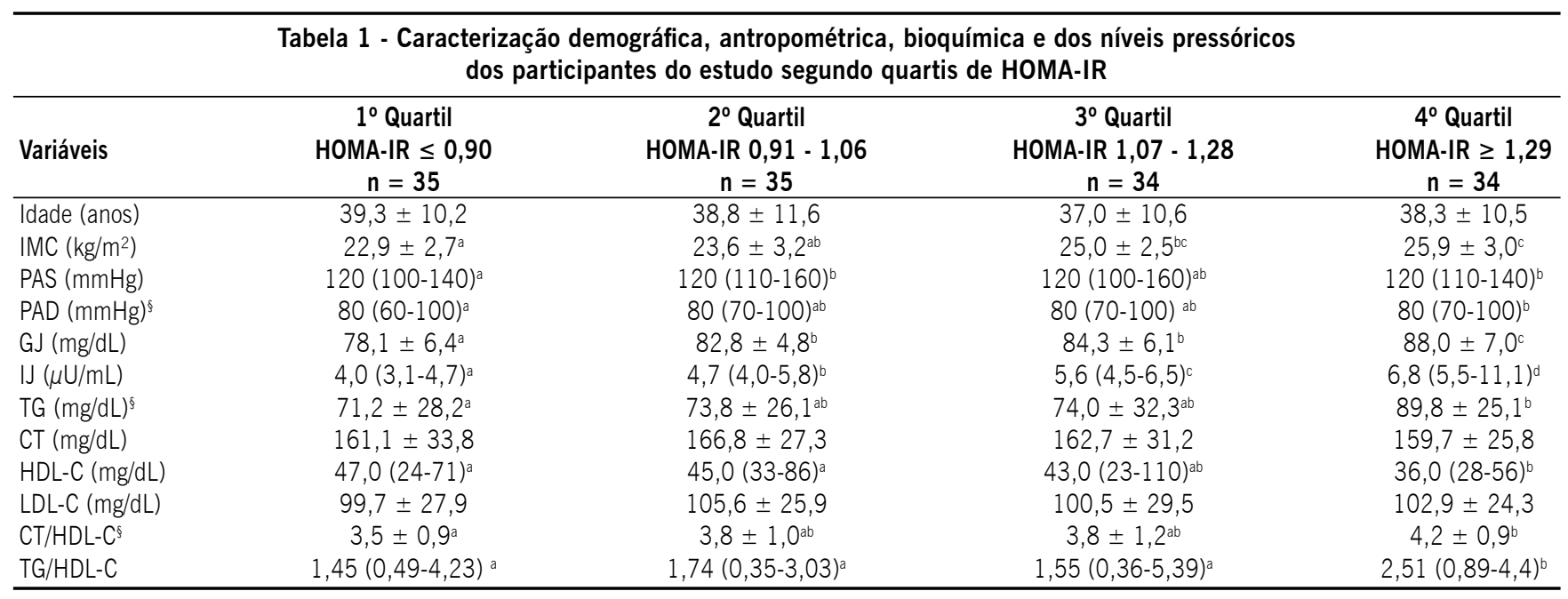

${ }^{8} \mathrm{p}<0,05 ; \mathrm{p}<0,01 ; \mathrm{p}<0,001$. ANOVA e teste post-hoc de Tuckey para as variáveis apresentadasem média \pm desvio padrão. Teste de Kruskall Wallis e teste post-hoc de Dunn's para as variáveis apresentadas em mediana (valores mínimos-máximos). Comparação entre colunas: letras iguais indicam valores iguais e letras diferentes indicam diferença estatística significante entre os valores. $\mathrm{CT}=$ colesterol total, GJ = glicemia de jejum, IJ = insulinemia de jejum, IMC $=$ índice de massa corporal, PAS = pressão arterial sistólica, $\mathrm{PAD}=$ pressão arterial diastólica, $\mathrm{CT} / \mathrm{HDL}-\mathrm{C}=$ relação colesterol total/HDL-C, TG/HDL-C $=$ relação triglicérides/HDL-C, TG $=$ triglicérides .

\section{Figura 1 - Curva ROC para os indicadores bioquímicos do perfil} lipídico plasmático na avaliação de níveis de HOMA-IR

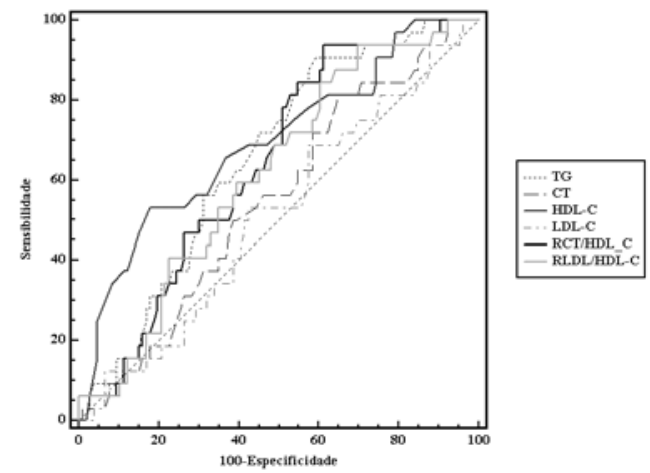

$\mathrm{CT}=$ colesterol total, CT/HDL-C = relação colesterol total/HDL-C, TG/HDL-C = relação triglicérides/HDL-C, TG = triglicérides.

para os valores das variáveis bioquímicas estudadas, mesmo em se tratando de indivíduos saudáveis.

Em concordância, McLaughlin et al. ${ }^{5}$ avaliaram 258 indivíduos (127 homens) caucasianos não- diabéticos, normotensos, com excesso de peso corporal e identificaram que a relação TG/HDL-C e os TG apresentaram melhor eficácia em avaliar a presença de RI comparados ao $\mathrm{HDL}-\mathrm{C}$, ao CT e à relação $\mathrm{CT} /$ HDL-C. Hannon et al. ${ }^{7}$ identificaram a relação TG-HDL-C e os TG como os melhores indicadores de RI comparados ao LDL-C, ao CT e ao HDL-C em adolescentes.

A melhor eficácia encontrada para a relação TG/HDL-C na avaliação dos níveis mais elevados de HOMA-IR reflete o perfil dislipidêmico característico dos indivíduos com RI, que tem sido explicado por alterações metabólicas causadas pela sinalização

\begin{tabular}{lc}
\hline & $\begin{array}{c}\text { Tabela 2 - Áreas abaixo da curva ROC para os } \\
\text { indicadores bioquímicos do perfil lipídico } \\
\text { plasmático segundo o índice HOMA-IR }\end{array}$ \\
\hline Variáveis & Área \pm EP (IC 95\%) \\
\hline TG & $0,674 \pm 0,048(0,579-0,768)$ \\
CT & $0,445 \pm 0,053(0,340-0,550)$ \\
HDL-C & $0,716 \pm 0,052(0,615-0,817)$ \\
LDL-C & $0,489 \pm 0,055(0,381-0,597)$ \\
CT/HDL-C & $0,655 \pm 0,050(0,558-0,753)$ \\
TG/HDL-C & $0,724 \pm 0,046(0,633-0,815)$ \\
\hline
\end{tabular}

$p<0,01 ; p<0,001 . C T=$ colesterol total, CT/HDL-C = relação colesterol total/ HDL-C, TG/HDL-C = relação triglicérides/HDL-C, TG = triglicérides.

deficiente da insulina, especialmente no tecido adiposo ${ }^{1}$. Na RI, a supressão prejudicada da lipólise resulta em liberação aumentada de ácidos graxos livres na corrente sanguínea e em aumento da oferta de ácidos graxos ao fígado, com subsequente estímulo para a síntese de triglicérides e de VLDL-C. Em condições normais, a sinalização da insulina leva à degradação da apolipoproteína B, o que não ocorre de forma adequada nos estados de RI. A RI reduz a atividade da lipase lipoprotéica, mediador do clearance de VLDL-C, o que também contribui, em menor proporção, para a elevação dos TG. Assim, a combinação entre maior síntese de VLDL-C, menor degradação de apoB e menor atividade da lipase lipoprotéica explica a hipertrigliceridemia característica da RI. Na RI, concentrações aumentadas de partículas de VLDL-C ricas em triglicérides contribuem para o metabolismo anormal do HDL-C. A proteína de transferência de ésteres de colesterol medeia a troca de ésteres de colesterol do HDL-C com os TG da VLDL-C, resultando em HDLs-C ricas em TG e VLDLs ricas em ésteres de colesterol. O aumento da concentração de TG na partícula de HDL-C torna-a um excelente substrato para a lipase hepática, contribuindo para a redução dos níveis de HDL-C ${ }^{20,21}$. 
Do ponto de vista fisiopatológico e de acordo com os resultados do presente estudo, a relação TG/HDL-C parece ser mais eficiente na avaliação da presença de RI do que o HDL-C isolado. Além do mais, trabalhos demonstraram que as concentrações plasmáticas de TG e de HDL-C são preditoras independentes de $\mathrm{RI}$ e de doença cardiovascular 22,23 . Dessa forma, acreditamos que a utilização da relação TG/HDL-C, ao invés da medida isolada do HDL-C, seja preferível, embora a aplicação desta última seja mais simples na prática.

Embora o índice HOMA-IR não seja a técnica padrão-ouro para avaliação da RI, ele representa um método adequado para estudos populacionais pela forte correlação demonstrada com a técnica de clamp euglicêmico hiperinsulinêmico ${ }^{3,4}$. Além do mais, foi utilizado um ensaio para insulina com ausência de reação cruzada com pró-insulina, o que garante maior confiabilidade das determinações plasmáticas. Apesar da praticidade na utilização do HOMA-IR na pesquisa, quando comparado ao clamp; na prática clínica, a determinação da insulinemia de jejum conta com as desvantagens da ausência de padronização entre os laboratórios, tanto no que diz respeito ao método quanto ao ensaio utilizado ${ }^{24}$. Em relação ao custo, são dosagens relativamente caras e indisponíveis em muitos serviços de saúde. Neste contexto, a determinação do perfil lipídico conta com a vantagem da melhor padronização entre os laboratórios, com a maior facilidade de acesso aos serviços de saúde e menor custo.

Outro ponto importante diz respeito à inclusão no estudo de indivíduos normotensos, não hiperlipidêmicos e com tolerância normal à glicose, o que resultou, embora não propositalmente, em uma amostra composta por indivíduos com baixos níveis insulinêmicos, e, consequentemente, níveis de HOMA-IR relativamente baixos, o que pode ter enfraquecido as correlações. Provavelmente, a avaliação de níveis mais altos de RI deverá necessitar de níveis mais elevados para a relação TG/HDL-C, HDL-C e TG. No entanto, ressalta-se, que mesmo em se tratando de uma amostra composta apenas por indivíduos saudáveis, foram identificados aumentos nos níveis pressóricos, no IMC, nos níveis de TG e redução de HDL-C em função do aumento do índice HOMA-IR. Em outras palavras, o presente estudo identificou tal comportamento em pessoas consideradas saudáveis pelos pontos de corte atualmente recomendados por consensos de abrangência nacional e internacional, demonstrando que essas relações coexistem muito antes que determinada morbidade ocorra.

\section{Conclusão}

Em conclusão, a relação TG/HDL-C foi o melhor indicador bioquímico do perfil lipídico na avaliação de níveis mais elevados de HOMA-IR devido ao melhor desempenho nas análises ROC e de correlação. Sugerimos a realização dessas investigações em outros extratos da população, incluindo mulheres e indivíduos de outras faixas etárias. Com o propósito de viabilizar a utilização desse indicador na prática, o comportamento da relação TG/ HDL-C deve ser avaliado em níveis mais altos de TG séricos, para possibilitar a determinação de pontos de corte. Estes resultados sugerem a aplicação da relação TG/HDL-C para a avaliação da RI como instrumento alternativo, de mais fácil acesso na prática clínica, para intervenções de caráter preventivo de doenças na população brasileira.

\section{Agradecimentos}

Agradecemos ao Conselho Nacional de Desenvolvimento Científico e Tecnológico (CNPq) e à Fundação de Amparo à Pesquisa do Estado de Minas Gerais (FAPEMIG) pelo apoio financeiro e ao Laboratório de Investigação em Metabolismo e Diabetes (LIMED) - UNICAMP pela parceria científica.

\section{Suporte Financeiro}

FAPEMIG e CNPq

\section{Conflito de interesse: não há}

\section{SUMMARY}

Plasmatic Lipid PRofile INDicators Related to INSUlin Resistance

PurPose. To investigate the effectiveness of biochemical indicators from the plasmatic lipid profile to identify the insulin resistance (IR), assessed by the HOMA-IR index (Homeostasis Model Assessment - Insulin Resistance).

Methods. 138 healthy men (20-59 years) were evaluated. The lipid profile biochemical indicators analyzed were the following: triglycerides (TG), total cholesterol (TC), HDL-C, $L D L-C$, and TC/HDL-C and TG/HDL-C ratios. The percentile 75 was considered as the cut-off point for IR. Statistical analysis consisted of Spearman correlation coefficient, ROC curves and calculation of the areas under the curve (AUC).

RESULT. The TG/HDL-C ratio showed the strongest correlation and the greatest $A \cup C(r=0.334$ and $A \cup C=0.724 \pm 0.046$, $p<0.001)$ respectively, followed by the HDL-C $(r=-0.313$ and $A U C=0.716 \pm 0.052, p<0.01)$, the TG $(r=0.261$ and $A U C=0.048 \pm 0.674, p<0.01)$ and CT/HDL-C ratio $(r$ $=0.259$ and $A U C=0.655 \pm 0.05, p<0.01)$. Results for $T C$ and LDL-C showed no statistical significant $(p>0.05)$.

CONCLUSION. The TG/HDL-C ratio showed the greatest ability to identify $I R$, proved to be an alternative and easy access instrument to assess IR in clinical practice, therefore providing diseases prevention for the adult male population. [Rev Assoc Med Bras 2009; 55(3): 342-6]

KEY WORDS: Insulin resistance. HOMA. Lipoproteins. Triglycerides.

\section{REFERÊNCIAS}

1. Mlinar B, Marc J, Janez A, Pfeifer M. Molecular mechanisms of insulin resistance and associated diseases. Clin Chim Acta. 2007;375:20-35.

2. Lee S, Gungor N, Bacha F, Arslanian S. Insulin resistance: link to the components of the metabolic syndrome and biomarkers of endothelial dysfunction in youth. Diabetes Care. 2007;30:2091-7.

3. Geloneze B, Tambascia MA. Avaliação laboratorial e diagnóstico de resistência insulínica. Arq Bras Endocrinol Metabol. 2006;50:208-15.

4. Vasques ACJ, Rosado LEFPL, Alfenas RCG, Geloneze B. Análise crítica do uso dos índices do Homeostasis Model Assessment (HOMA) na avaliação da resistência à insulina e capacidade funcional das células-b pancreáticas. Arq Bras Endocrinol Metabol. 2008;52:32-9.

5. McLaughlin T, Abbasi F, Cheal K, Chu J, Lamendola C, Reaven G. Use of metabolic markers to identify overweight individuals who are insulin resistant. Ann Intern Med. 2003;139:802-9.

6. McLaughlin T, Reaven G, Abbasi F, Lamendola C, Saad M, Waters D, et al. Is there a simple way to identify insulin-resistant individuals at increased risk of cardiovascular disease? Am J Cardiol. 2005;96:399-404.

7. Hannon TS, Bacha F, Lee SJ, Janosky J, Arslanian SA. Use of markers of dyslipidemia to identify overweight youth with insulin resistance. Pediatr Diabetes. 2006; 7:260-6.

8. Sumner AE, Cowie CC. Ethnic differences in the ability of triglyceride levels to identify insulin resistance. Atherosclerosis. 2008;196:696-703. 
9. Palaniappan LP, Kwan AC, Abbasi F, Lamendola C, McLaughlin TL, Reaven GM. Lipoprotein abnormalities are associated with insulin resistance in South Asian Indian women. Metabolism. 2007;56:899-904.

10. Sumner AE, Finley KB, Genovese DJ, Criqui MH, Boston RC. Fasting triglyceride and the triglyceride-HDL cholesterol ratio are not markers of insulin resistance in African Americans. Arch Intern Med. 2005;165:1395-400.

11. Pimenta JR, Zuccherato LW, Debes AA, Maselli L, Soares RP, Moura-Neto RS, et al. Color and genomic ancestry in Brazilians: a study with forensic microsatellites. Hum Hered. 2006;62:190-5.

12. Sociedade Brasileira de Cardiologia, Sociedade Brasileira de Nefrologia. V Diretrizes Brasileiras de Hipertensão Arterial. Arq Bras Cardiol. 2007·89:e24-e79.

13. Jelliffe DB. Evaluación del estado nutrición de la comunidad. Ginebra: Organización Mundial de La Salud, 1968. p.166.

14. World Health Organization. (WHO). Obesity: preventing and managing the global epidemic. Report of a WHO Consultation. Geneva: World Health Organization; 2000.

15. Friedewald WT, Levy RI, Fredrickson DS. Estimation of the concentration of low-density lipoprotein cholesterol in plasma, without use of the preparative ultracentrifuge. Clin Chem. 1972;18:499-502.

16. Matthews DR, Hosker JP, Rudenski AS, Naylor BA, Treacher DF, Turner RC. Homeostasis model assessment: insulin resistance and beta-cell function from fasting plasma glucose and insulin concentrations in man. Diabetologia. 1985;28:412-9.

17. Hanley JA, McNeil BJ. A method of comparing the areas under receiver operating characteristic curves derived from the same cases. Radiology. 1983; 148:839-43.
18. Bonora E, Kiechl S, Willeit J, Oberhollenzer F, Egger G, Targher G, et al. Prevalence of insulin resistance in metabolic disorders: the Bruneck Study. Diabetes 1998;47:1643-9.

19. Oliveira EP, de Lima MD, de Souza ML. Metabolic syndrome, its phenotypes, and insulin resistance by HOMA-IR. Arq Bras Endocrinol Metabol. 2007;51:1506-15.

20. Shoelson SE, Lee J, Goldfine AB. Inflammation and insulin resistance. J Clin Invest 2006;116:1793-801.

21. Semenkovich CF. Insulin resistance and atherosclerosis. J Clin Invest. 2006;116:1813-22.

22. Zavaroni I, DallAglio E, Alpi O, Bruschi F, Bonora E, Pezzarossa A, et al. Evidence for an independent relationship between plasma insulin and concentration of high density lipoprotein cholesterol and triglyceride. Atherosclerosis. 1985;55:259-66.

23. Laws A, Reaven GM. Evidence for an independent relationship between insulin resistance and fasting plasma $\mathrm{HDL}$-cholesterol, triglyceride and insulin concentrations. J Intern Med. 1992;231:25-30.

24. Manley SE, Stratton IM, Clark PM, Luzio SD. Comparison of 11 human insulin assays: implications for clinical investigation and research. Clin Chem. 2007;53:922-32.

Artigo recebido: 28/07/08 Aceito para publicação: 21/01/09 